\title{
Energy calibration and gain correction of pixelated spectroscopic $x$-ray detectors using correlation optimised warping
}

DOI:

10.1088/1361-6501/28/1/017001

\section{Document Version}

Accepted author manuscript

Link to publication record in Manchester Research Explorer

Citation for published version (APA):

Egan, C., Scuffham, J. W., Veale, M. C., Wilson, MD., Seller, P., \& Cernik, R. (2016). Energy calibration and gain correction of pixelated spectroscopic $\mathrm{x}$-ray detectors using correlation optimised warping. Measurement Science and Technology. https://doi.org/10.1088/1361-6501/28/1/017001

Published in:

Measurement Science and Technology

\section{Citing this paper}

Please note that where the full-text provided on Manchester Research Explorer is the Author Accepted Manuscript or Proof version this may differ from the final Published version. If citing, it is advised that you check and use the publisher's definitive version.

\section{General rights}

Copyright and moral rights for the publications made accessible in the Research Explorer are retained by the authors and/or other copyright owners and it is a condition of accessing publications that users recognise and abide by the legal requirements associated with these rights.

\section{Takedown policy}

If you believe that this document breaches copyright please refer to the University of Manchester's Takedown Procedures [http://man.ac.uk/04Y6Bo] or contact uml.scholarlycommunications@manchester.ac.uk providing relevant details, so we can investigate your claim.

\section{OPEN ACCESS}




\title{
Energy calibration and gain correction of pixelated spectroscopic X-ray detectors using correlation optimised warping
}

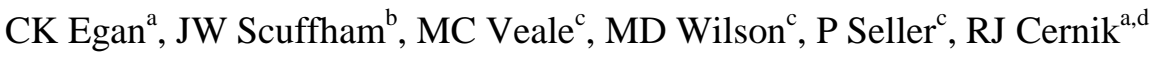 \\ ${ }^{a}$ Henry Moseley X-ray Imaging Facility, School of Materials, The University of Manchester, \\ Manchester, M13 9PL, UK \\ ${ }^{\mathrm{b}}$ Department of Nuclear Medicine, Royal Surrey County Hospital NHS Foundation Trust, Guildford, \\ Surrey, UK \\ ${ }^{c}$ Science and Technology Facilities Council, Rutherford Appleton Laboratory, Harwell Science and \\ Innovation Campus, Oxfordshire, UK \\ ${ }^{\mathrm{d}}$ ESRF, 71 Rue des Martyrs, Grenoble, France
}

\begin{abstract}
We describe the implementation of a reliable, robust and flexible gain correction and energy calibration algorithm for pixelated spectroscopic X-ray detectors. This algorithm uses a data processing method known as correlation optimised warping which aligns shifted datasets by means of a segmental linear stretching and compression of the spectral data in order to best correlate with a reference spectrum. We found the algorithm to be very robust against low-count spectroscopy, and was reliable in a range of different spectroscopic applications. Analysis of the integrated spectrum over all pixels for a Cerium K-alpha X-ray emission (at $34.72 \mathrm{keV}$ ) yielded a peak width of $2.45 \mathrm{keV}$ before alignment and $1.11 \mathrm{keV}$ after alignment. This compares favourably with the best in class pixel peak width of $0.76 \mathrm{keV}$ and the mean peak width for all pixels of $1.00 \mathrm{keV}$. We also found the algorithm to be more user friendly than other peak-search algorithms because there is less external input. A key advantage of this algorithm is that it requires no prior knowledge of the input spectral characteristics, shape or quality of the data. This therefore lends itself to being useful for in-line processing and potentially removes the need for a separate calibration standard (e.g. a radioactive source). This algorithm can be used for any system that simultaneously collects large numbers of spectral data - including multi-element detectors.
\end{abstract}

\section{Introduction}

Energy-resolving semiconductor detectors are extensively used for the quantitative study of the energy spectra from X-ray and gamma-ray sources. Applications include: elemental analysis in materials and geo-sciences; nuclear safety and environmental monitoring; nuclear medicine and astronomy. Increasingly, multi-element semiconductor detectors are being developed in order to improve count rates and sensitivities. These are finding applications in electron microscopes and at synchrotron radiation facilities to enable fast acquisitions and mapping capabilities [1,2]. 4-element silicon drift diode and 32-element monolithic germanium detectors are now widely available [3,4]. A 384 element silicon detector has also been reported [5]. Pixelated semiconductor detectors are also in development, these have the ability to measure X-ray spectra in a pixelated manner enabling the combination of imaging and spectroscopy. A 264x264 pixel silicon pnCCD detector has been developed [6] and a $400 \times 400$ pixel CdTe detector has also been reported [7]. These types of pixelated detectors can be applied to a variety of areas of research such as: biomedical science [8-10], materials and geological sciences [11-15], astronomy [16], cultural heritage[17] and security screening $[18,19]$ applications. 
An important data processing step for both multi-element and pixelated detectors is the energy calibration and gain correction of individual detector elements or pixels. This process enables the spectra produced from each pixel to be aligned on a common energy axis. Due to inhomogeneities between individual pixels arising from impurities and defects and/or manufacturing variations in electronic components, the inter-pixel spectral performance can vary widely. This can manifest itself either as variations in the gain, producing an apparent energy shift and offset, or as variations in the spectral performance through changes in the energy resolution, sensitivity or noise. Whilst the spectral performance of individual pixels cannot generally be improved by post-processing, corrections for gain variations can be achieved using a calibration procedure. This typically involves exposing the detector to a uniform flux of radiation with known specific characteristic emissions (e.g. from a radioactive source), applying an algorithm to identify these characteristic emissions in each pixel spectrum, and then aligning them on to a common axis. As the number of pixels in spectroscopic detectors increases, automated correction algorithms that can reliably and rapidly align large numbers of spectra will become increasingly important.

Many techniques for automated spectral calibration and gain correction have been published, generally involving least-squares fitting of an emission line with modified Gaussian functions representing the peak shape $[20,21]$. This is sometimes performed after prior convolution of the spectra to reduce background and emphasize the peaks [22]. A method based upon a peak search algorithm has also been reported which was found to be reliable for gamma-ray spectroscopic data [23].

Whilst these peak fitting or peak search algorithms have been shown to perform well for the applications described, there may be situations in which they may not be reliable, or even practical. For example, in the case of a detector that has a particularly pronounced inter-pixel gain variation (e.g. due to an inhomogeneous detector medium), the individual spectra may vary by so much that it becomes difficult to confidently identify the exact peak of interest using an automated algorithm that relies on some prior knowledge of the peak locations. Another potential scenario would be when there are no specific characteristic peaks to use as a calibration, for example when measuring the bremsstrahlung radiation emitted from an X-ray tube. A final example is the case of a pixelated detector that has a generally good spectral response, apart from a minority of poor responding pixels (say $<1 \%$ of the total). These pixels might show a range of responses, from completely inoperative, to reduced sensitivity or reduced energy resolution. These pixels could be easily masked out and disregarded, however this is potentially wasteful since many may still contain some adequate spectral information.

The above examples highlight the potential shortcomings of simple peak-search algorithms for energy calibration and gain correction of pixelated spectroscopic detectors. Herein, we describe the implementation of a reliable, robust and flexible gain correction and energy calibration algorithm that can be applied to a variety of data types and pixel detector responses. This algorithm uses a data processing method known as correlation optimised warping (COW). COW aligns shifted datasets by means of a segmental linear stretching and compression of the spectral data in order to best correlate with a reference spectrum. This is achieved by dividing the un-aligned data into a user specified number of segments. Each segment may be warped to a smaller or greater length using linear interpolation. A second parameter, called the slack, is defined so as to limit the movement of the segment boundary and as such limit to a finite (and calculable) number of possible combinations. Determining the optimal alignment is now a question of finding the optimal combination of warpings of the segments, where no segment may be warped by more than the slack length. This is achieved using a correlation coefficient and by examining all possible combinations section by section using 
dynamic programming. The fundamental principles of COW are well developed: The algorithm was introduced by Nielsen et al. [24] as a method to correct for shifts in discrete data signals and has principally been used to align chromatographic data [25] but has found multiple applications since $[26,27]$. The main advantage of this algorithm is its semi-automatic nature, enabling large numbers of spectra to be processed with very little external user input. This therefore lends itself to be highly useful for calibrating and aligning data collected from pixelated spectroscopic X-ray detectors, whereby thousands of spectra need to be individually aligned and calibrated.

\section{Methods}

\section{Detector}

The spectroscopic X-ray detector used in this work is the HEXITEC detector [28]. This consists of a 1 $\mathrm{mm}$ thick CdTe single crystal detector $\left(20 \times 20 \mathrm{~mm}^{2}\right)$ bump-bonded to a large area ASIC packaged with a high performance data acquisition system. The detector has $80 \times 80$ pixels on a $250 \mu \mathrm{m}$ pitch with an energy resolution of $800 \mathrm{eV}$ at $59.5 \mathrm{keV}$ and $1500 \mathrm{eV}$ at $141 \mathrm{keV}$ [28]. During operation each photon event has its energy, pixel position and the frame in which it occurs recorded. Events are processed and histograms are formed according to measured energy into $0.25 \mathrm{keV}$ wide bins. Between 400 - 1000 bins are typically used, depending on the maximum X-ray energy. Normally, during this process, a correction is employed to deal with so-called charge sharing, in which the energy deposited by a photon spreads between two or more pixels, which appear to be measured as multiple lower energy photon measurements on neighbouring pixels [29]. When the flux is sufficiently low it is possible to identify these shared events and apply a correction. In this paper, we use charge sharing discrimination, where all shared events are deleted.

In order to fully test the performance of the COW algorithm, we have examined three different types of spectroscopic data: X-ray fluorescence (XRF), gamma-ray spectroscopy and X-ray spectroscopy. Each dataset was collected using different HEXITEC detector units.

\section{$\underline{X-r a y ~ f l u o r e s c e n c e ~ d a t a ~}$}

The XRF data was collected by illuminating a series of materials containing heavy elements ( $\mathrm{Pb}$ foil, Ta foil and Cerium oxide powder) with a laboratory X-ray tube $(120 \mathrm{kV}, 300 \mu \mathrm{A}$, tungsten target) and collecting the emission signal for a total exposure time of 5 minutes.

\section{$\underline{\text { Gamma-ray data }}$}

The gamma-ray spectra were collected from a flood source, containing a mixture of $29 \mathrm{MBq}$ of Ga-67 gallium citrate, $30 \mathrm{MBq}$ of Tc-99m sodium pertechnetate, $55 \mathrm{MBq}$ of I-123 sodium iodide and pure water. These radionuclides are common in clinical nuclear medicine applications and were chosen to cover a broad spectral range. Data were acquired over 40 minutes to collect a sufficient number of events to conduct analysis of the spectra collected by each pixel.

\section{$\underline{\text { Spectroscopic X-ray data }}$}

The absorption spectra were measured by directly illuminating the detector with X-rays from a laboratory microfocus X-ray tube $(70 \mathrm{kV}, 10 \mu \mathrm{A}$, tungsten target) filtered by $1 \mathrm{~mm}$ of copper, with a total exposure time of 1 minute. 
Algorithm

The following was implemented using MATLAB version 8.3 (R2014a) and the COW MATLAB toolbox which can be found at [30], as developed by Tomasi et al. [25]. There are two initial parameters that need to be defined in order to implement the COW algorithm: segment length and slack. The choice of these input parameters may influence the algorithm performance as a whole. Therefore, the COW toolbox includes an automated optimisation routine which estimates the most appropriate values for these parameters based upon the raw data, or a portion of the raw data [31]. Depending on the type (e.g. X-ray fluorescence, gamma-ray or X-ray absorption spectra) and quality (e.g. signal-to-noise ratio) of the data, the segment length and slack parameters found by this algorithm may vary, but are largely related to the total number of channels in the spectrum. For the data presented below, using this automated optimisation routine we obtained values for the segment length to be in the range of $15-40$, and slack in the range of $2-15$. We also developed an independent test algorithm which measured the full width at half maximum (FWHM) of a gamma-ray photopeak after summing all pixel spectra. This gives a measure of the overall performance of the COW algorithm, as any mis-aligned pixel spectra will lead to a degraded FWHM. By iteratively varying the values for segment length and slack, our independent algorithm obtained optimum parameters of segment length $=20$ and slack $=10$. Therefore for data which is 400 channels in length, this is split into 20 segments, with boundaries allowed to shift by $+/-10$ channels. In practice, we found the warping parameters returned by the COW algorithm to be relatively insensitive to the segment length and slack, provided they were in the ranges obtained by the optimisation routine provided with the COW toolbox. As such, we used the parameters obtained by our independent optimisation algorithm for all subsequent analysis.

After choosing the appropriate values for segment length and slack, the COW algorithm can then be implemented. This is achieved by moving the segment boundaries (within the confines of the slack parameter) whilst correlating against a reference spectrum. This is performed for every pixel spectrum. The output of this algorithm is the segment starting points for each pixel - these we call the 'warping values'. These values are then input into a separate application which performs linear interpolation over the segment lengths so as to stretch and shift the spectra. The final result should be well aligned spectra. A snippet of the MATLAB code to implement this is shown in Figure 1. 


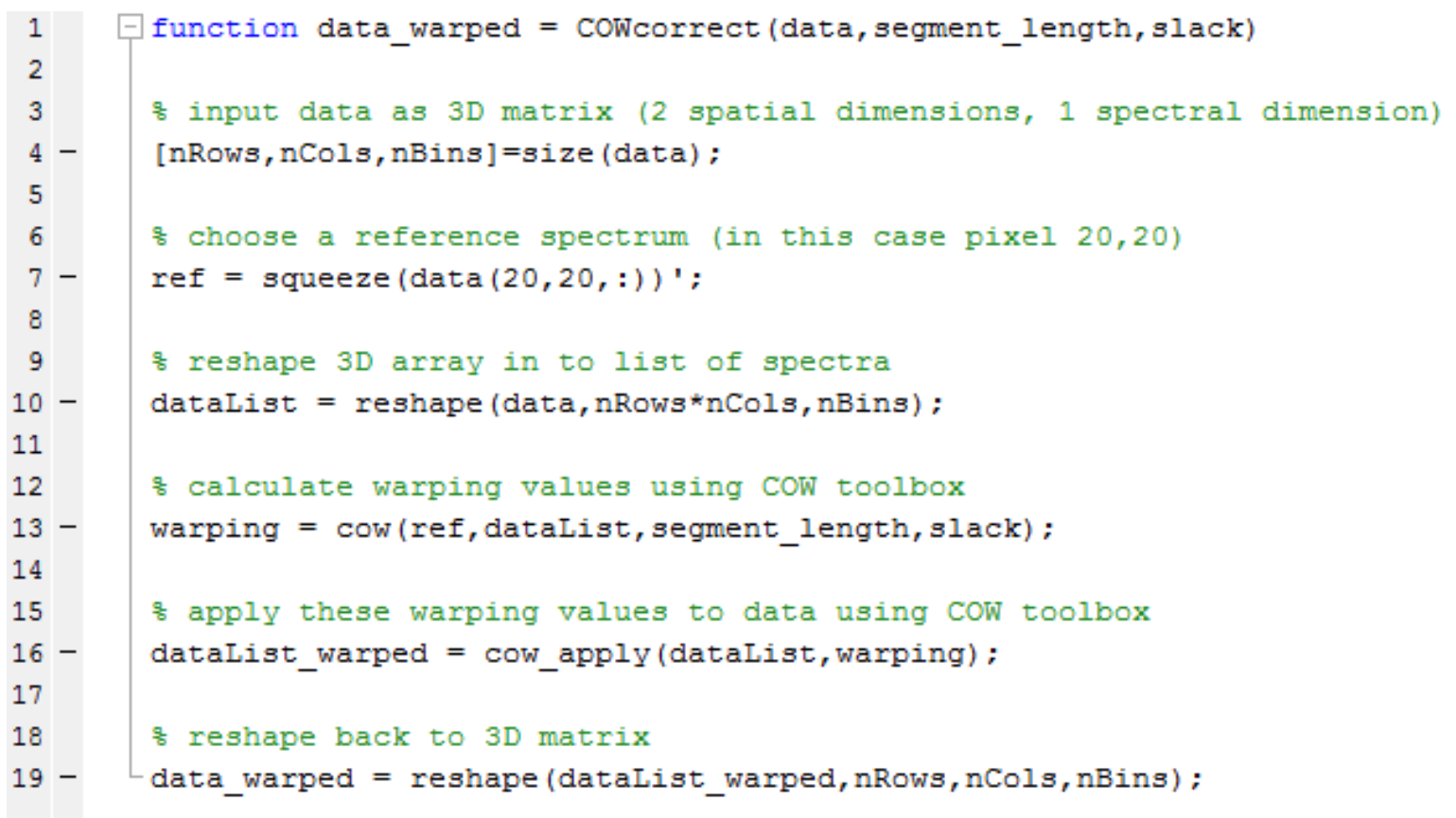

Figure 1: MATLAB code snippet used to execute the COW algorithm. The functions 'cow' and 'cow_apply' are both from the COW MATLAB toolbox.

An important aspect is the choice of reference spectrum. This has a strong influence on the performance of the COW algorithm since all other spectra are correlated and warped against this reference, so it is appropriate to pick a reference that shows a 'normal' behaviour. Therefore, the reference spectrum should have gain value comparable to the average across the entire detector, and it should also have good spectral performance (low noise, high energy resolution). To avoid subjective bias in the selection of a reference spectrum, we used four separate randomly chosen reference spectra from known "good" detector pixels, and calculated the warping values for each one. We then took the average figures from these four, and used these as the final warping values. We found that this had the advantage of removing noise that was apparent in the total integrated spectrum when using lower quality reference spectra, whilst at the same time negating any user input bias of the over the choice of the reference spectrum.

\section{Results \& Discussion}

\section{$\underline{X-r a y ~ f l u o r e s c e n c e ~ s p e c t r o s c o p y ~}$}

Figure 2a shows two randomly selected pixel spectra before and after correction using COW. Before correction, the spectra are poorly aligned in the energy axis due to variations in inter-pixel gain. After correction (Figure $2 b$ ), the spectra are much more closely aligned, allowing the energy axis to be calibrated using the known peak energies based on any one pixel spectrum. Figure $2 \mathrm{c}$ shows a calibration plot using some of the XRF peaks. A least-squares linear fit yields the energy calibration $y$ $=0.231[\mathrm{keV} /$ channel] $x+1.59[\mathrm{keV}]$, where $\mathrm{x}$ is the uncalibrated axis with units of channel number and $\mathrm{y}$ is the calibrated axis with units of $\mathrm{keV}$. The norm of the residuals for this fit was found to be 0.278. An advantage of calibrating in this fashion is that a peak search algorithm only has to be utilised once, whereas for a pixel-wise method, a peak search has to be applied to every individual spectrum. In practice, this can be highly computationally intensive. 

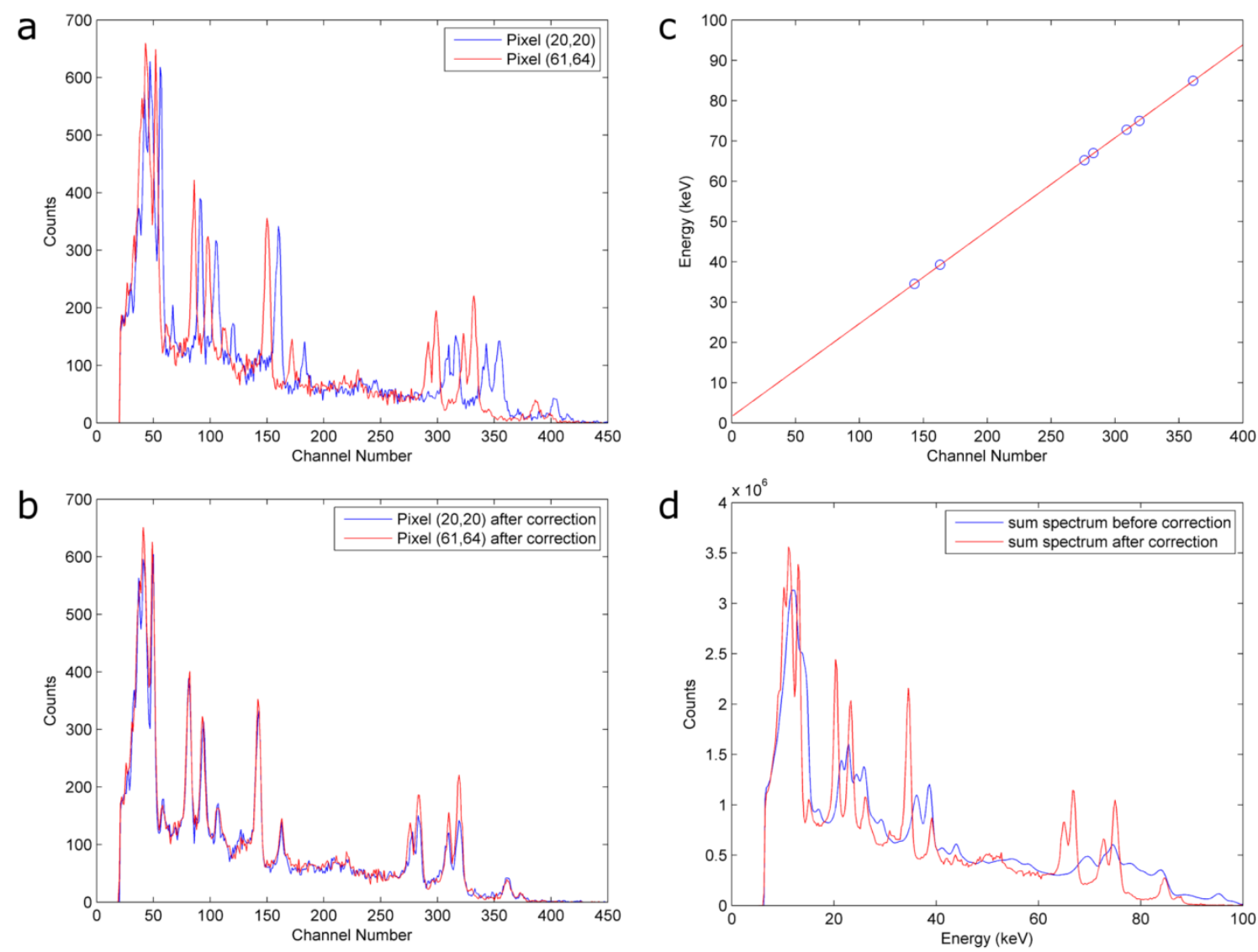

Figure 2: a) XRF data with two randomly selected pixel spectra showing a typical gain variation. b) The same two spectra after alignment using COW. c) Energy calibration plot from selected emission peaks. d) Total sum spectrum over all pixels before and after calibration.

A useful method for characterising the performance of the algorithm is to look at the total spectrum summed over all the pixels. This spectrum, without correction, would exhibit broad or overlapping peaks due to the random shift of each pixel spectrum along the energy axis. In contrast, a wellcorrected dataset would exhibit narrow peaks since all pixel spectra should be well aligned. Figure $2 \mathrm{~d}$ shows the integrated spectrum before and after energy scale correction using COW. Before calibration, it is very difficult to identify the specific peaks in the spectrum, due to the degraded spectral resolution caused by mis-aligned pixel spectra. The uncorrected summed spectra exhibit a doublet peak splitting, resulting from a bimodal distribution of gain factors across the detector. This could be due to the gain being affected in one area of the detector, for example due to poor bonding between detector and ASIC, potentially introducing a resistive or capacitive coupling. After correction, the total sum spectrum shows clean narrow peaks and resembles the shape of a single pixel spectrum. Fitting a Gaussian function to the Ce Ka peak (@34.7 keV) in the total sum spectrum obtained a FWHM of $2.45 \mathrm{keV}$ before alignment and $1.11 \mathrm{keV}$ after alignment. This compares favourably with the best in class pixel FWHM of $0.76 \mathrm{keV}$ and the mean FWHM for all pixels of 1.00 $\mathrm{keV}$. Typically $1 \%$ of pixels show some non-uniformities due to crystal defects, like inclusions. These manifest as broadening of the spectral lines or distortion of the shape of the spectrum (dependant on the depth of the defect in the crystal). Figure 3 shows how the COW algorithm handles these types of defective pixel spectra. Four separate spectra showing a variety of spectral responses are shown, all after alignment using COW. Pixel $(66,42)$ represents a good quality spectrum as a reference. Pixel 
$(38,40)$ shows poor spectral response at high energy, but with otherwise narrow peaks at low energy. Pixel $(76,1)$ shows good sensitivity but significant peak broadening at all energies. Pixel $(35,47)$ shows a generally poor response throughout the spectral range. What is evident is how the COW algorithm does a good job at aligning these poor quality spectra. This would otherwise be difficult or impossible using peak-search algorithms. The advantage of this approach is that useful spectral information can still be extracted, even for poor quality pixels. These pixels would otherwise have to have been disregarded using a peak-search algorithm, due to uncertainty on peak position finding.

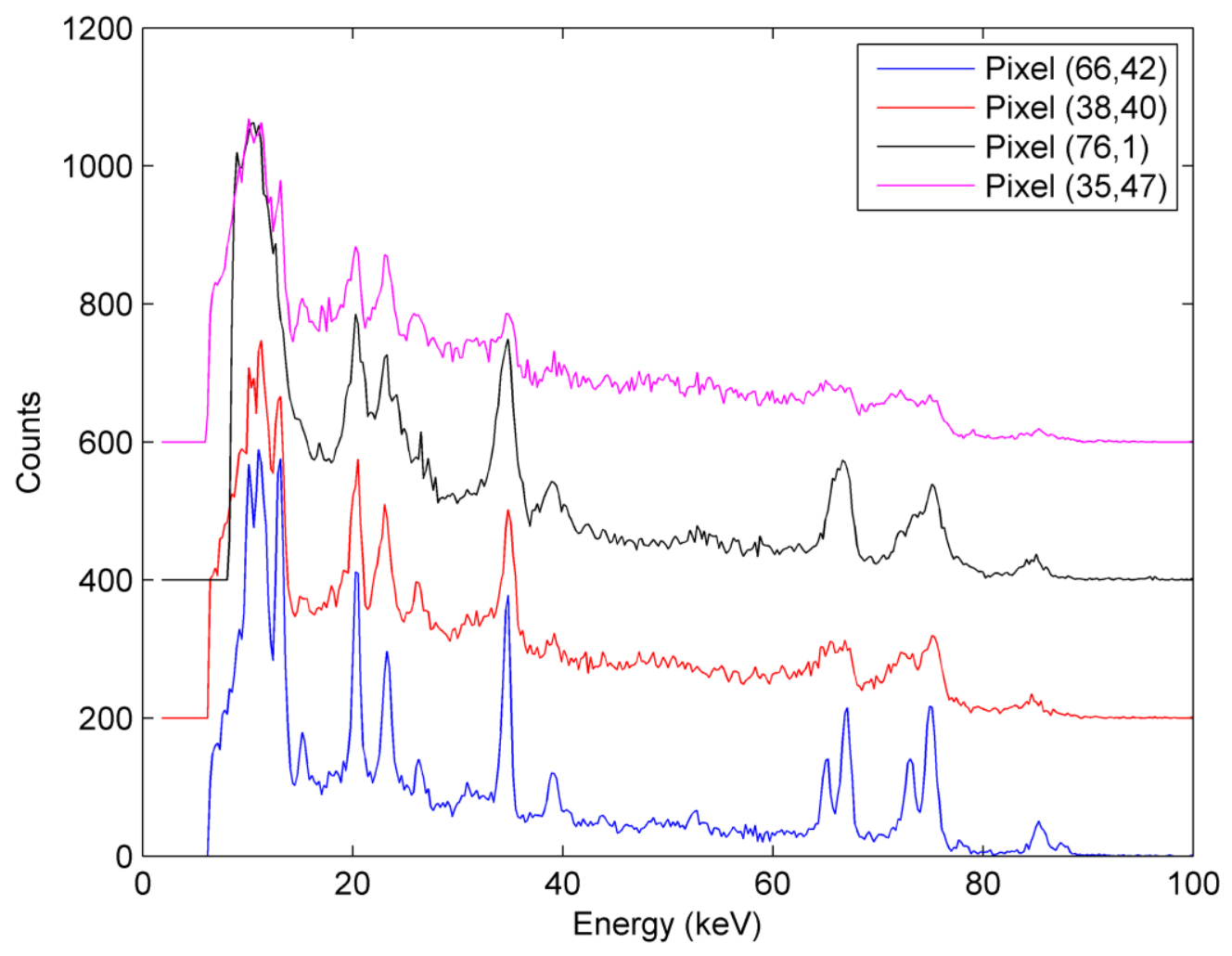

Figure 3: Four separate pixel spectra (offset vertically for clarity) showing a variety of spectral responses, all after alignment using COW. Pixel $(66,42)$ represents a good quality spectrum as a reference. Pixel $(38,40)$ shows poor spectral response at high energy. Pixel $(76,1)$ shows good sensitivity but significant peak broadening. Pixel $(35,47)$ shows a generally poor response throughout the spectral range. What is evident is that the COW algorithm does a good job at aligning these poor quality spectra.

\section{Spectroscopic X-ray imaging}

We have also tested the same algorithms on spectra collected directly from a laboratory tungsten target microfocus $\mathrm{X}$-ray source. This data represents a more challenging situation for a peak-search algorithm since there are few identifier peaks that can be used to align the spectra and the signal-tonoise ratio is relatively poor due to low number of counts. Figure 4 shows the results before and after applying the correction. In these spectra, the two peaks at channel numbers of about 100 and 115 are from $\mathrm{Cd}$ and $\mathrm{Te} \mathrm{K}$ line emissions. These are secondary emissions inside the detector, stimulated from the incident radiation and are self-absorbed. The main contribution to the spectra is from Bremstrallung radiation producing the broadband polychromatic emission profile. Before correction, the spectral features in each pixel are seen to be shifted relative to one another due to inter-pixel gain variations, as expected. After correction, it can be seen that the spectral features become well aligned 
(Figure 4b). In order to calibrate the energy axis, we used the end-point energies at different tube voltages $(40 \mathrm{kV}, 50 \mathrm{kV}, 60 \mathrm{kV}, 70 \mathrm{kV}$ and $80 \mathrm{kV})$ as the reference signal. The end-point energy is found by plotting the measured X-ray intensity (after summing over all pixels) on a logarithmic scale. Straight lines extrapolating the background signal and X-ray signal are drawn, the intersection point is used as the end-point. These values are plotted on a calibration curve as shown in Figure 4c. A leastsquares fit of a linear equation yields the energy calibration $y=0.207$ [keV/channel] $x+1.90[\mathrm{keV}]$, where $\mathrm{x}$ is the uncalibrated axis with units of channel number and $\mathrm{y}$ is the calibrated axis with units of $\mathrm{keV}$. The norm of the residuals for this fit was found to be 0.31 . We have again used the spectrum integrated over all pixels as a measure of the quality of the correction procedure, as shown in Figure $4 \mathrm{~d}$. It can be seen that the corrected spectrum shows narrow aligned peaks for the detector selfemission lines. There is some modification of the spectral shape for the bremsstrahlung emission, but otherwise there is very little change in the spectral response. This indicates that the gain variation was already very uniform for this detector prior to calibration.
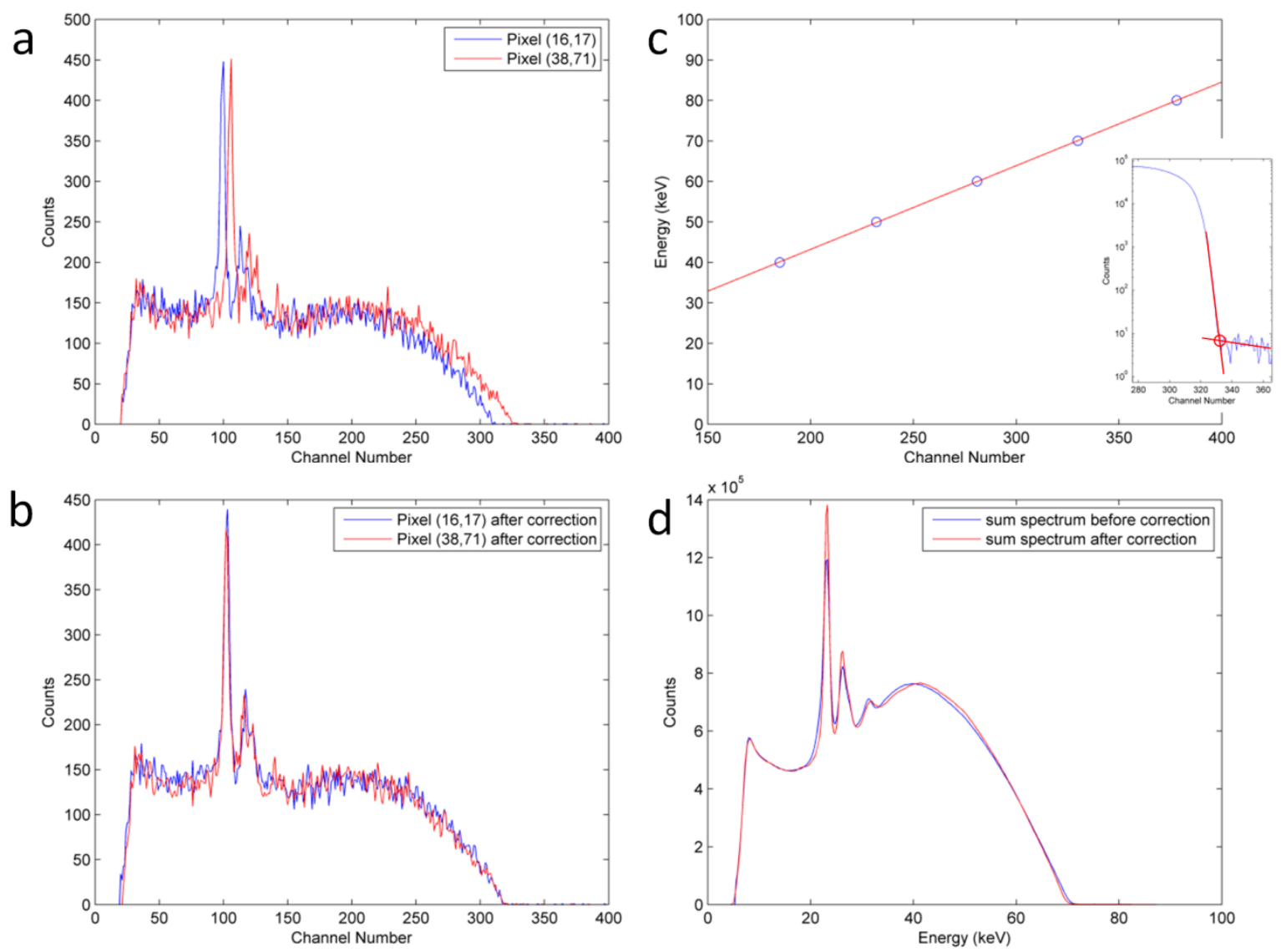

Figure 4: a) Spectra from a microfocus $\mathrm{X}$-ray tube $(70 \mathrm{kV})$ showing two randomly selected pixels with typical gain variations. b) The same pixels after alignment using COW. c) Calibration plot using the end-point energy as the reference signal for $40 \mathrm{kV}, 50 \mathrm{kV}, 60 \mathrm{kV}, 70$ $\mathrm{kV}$ and $80 \mathrm{kV}$. The inset depicts how the end-point energy is found through straight line intersects. d) Total sum spectrum over all pixels before and after alignment. 


\section{Gamma-ray spectroscopy}

Lastly we have tested COW on gamma-ray spectra collect from a series of common radionuclides. This type of data offers another challenging situation with the low-count spectra covering a wide energy range (up to 900 channels) with higher detector dynamic range. The results of applying the algorithm are shown in Figure 5. Figure 5a shows two randomly chosen pixel spectra exhibiting different gain values. After applying COW to this data, these pixel spectra become well aligned without affecting the overall spectral shape, as shown in Figure 5b. An energy calibration can now be performed by identifying individual gamma emission peaks, as shown in Figure 5c. A least-squares linear fit yields the energy calibration $y=0.214[\mathrm{keV} / \mathrm{channel}] x-0.40[\mathrm{keV}]$, where $\mathrm{x}$ is the uncalibrated axis with units of channel number and $\mathrm{y}$ is the calibrated axis with units of $\mathrm{keV}$. The norm of the residuals for this fit was found to be 3.66. Figure $5 \mathrm{~d}$ shows the summed spectrum over all pixels before and after application of the algorithm. A clear narrowing of peaks is observed, particularly at higher energies. Again, by extracting FWHM values for the I-123 peak (@159.0 keV) we obtained $3.14 \mathrm{keV}$ before alignment and $1.18 \mathrm{keV}$ after alignment. This compares favourably with the best in class pixel FWHM of $0.78 \mathrm{keV}$ and the mean FWHM for all pixels of $0.99 \mathrm{keV}$.

a

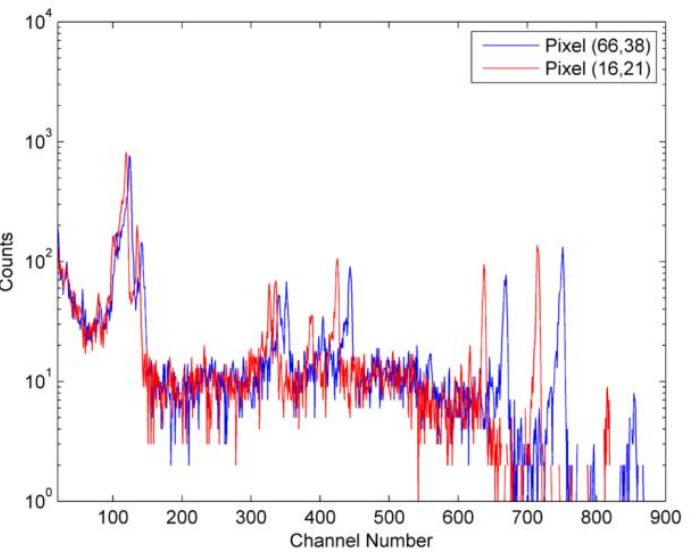

b

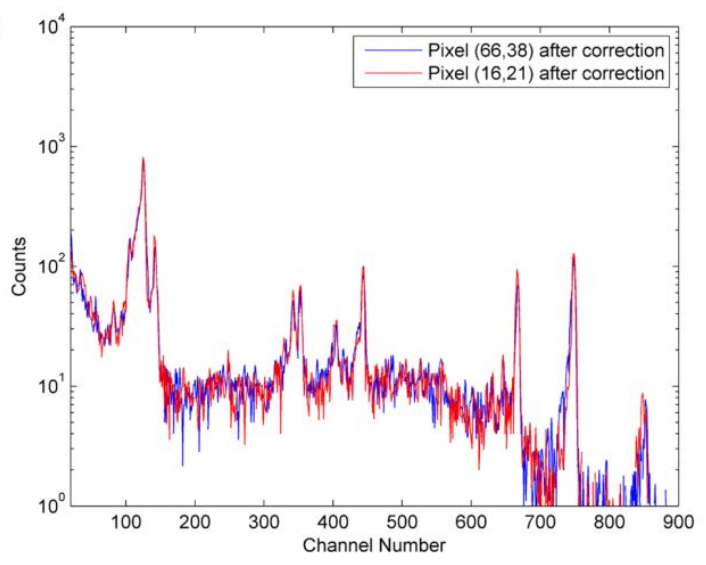

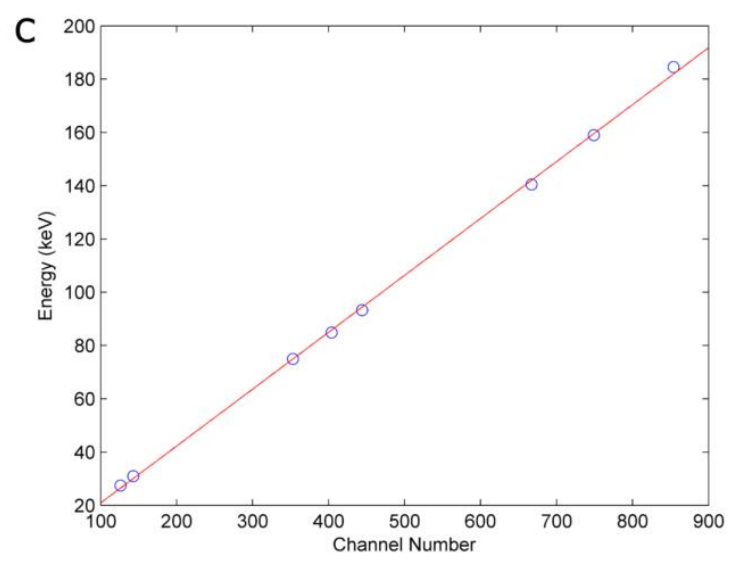

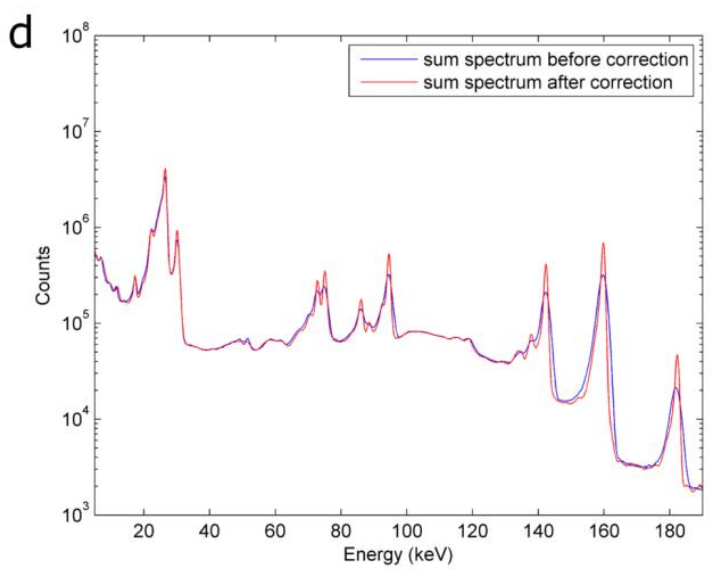

Figure 5: a) Gamma-ray spectra showing two randomly selected pixels with typical gain variations. b) The same two spectra after alignment using COW. c) Calibration plot from selected emission peaks. d) Total integrated spectrum before and after calibration. 


\section{Conclusions}

We have presented the application of correlated optimised warping to correct for gain variation in pixelated spectroscopic X-ray detectors. We found the algorithm to be very robust against low-count spectroscopy, and was reliable in a range of different spectroscopic applications. It is also easier to use than other peak-search algorithms because there is less user input. A key advantage of this algorithm is that it requires no prior knowledge of the input spectral characteristics, shape or quality of the data. This therefore lends itself to being useful for in-line processing and potentially removes the need for a separate calibration standard (e.g. a radioactive source). This algorithm can be used for any system that simultaneously collects large numbers of spectral data - including multi-element detectors.

\section{Acknowledgements}

The authors would like to acknowledge the following EPSRC grants: EP/F007906; EP/F001452; EP/I02249X; and EP/H046577/1. We are also grateful for funding from The Leverhulme Trust.

\section{References}

1. Ryan, C., et al., Elemental X-ray imaging using the Maia detector array: The benefits and challenges of large solid-angle. Nuclear Instruments and Methods in Physics Research Section A: Accelerators, Spectrometers, Detectors and Associated Equipment, 2010. 619(1): p. 37-43.

2. Schlossmacher, P., D. Klenov, B. Freitag, and H. Von Harrach, Enhanced detection sensitivity with a new windowless XEDS system for AEM based on silicon drift detector technology. Microscopy today, 2010. 18(04): p. 14-20.

3. Hitachi. http://www.hitachi-hightech.com/hhs-us/product_detail/?pn=ana-vortex-me4.

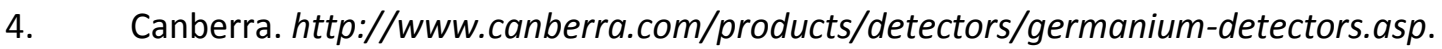

5. Kirkham, R., et al. The Maia spectroscopy detector system: engineering for integrated pulse capture, low-latency scanning and real-time processing. in American Institute of Physics Conference Proceedings. 2010. Brookhaven National Laboratory (BNL) National Synchrotron Light Source.

6. Ordavo, I., et al., A new pnCCD-based color X-ray camera for fast spatial and energy-resolved measurements. Nuclear Instruments \& Methods in Physics Research Section a-Accelerators Spectrometers Detectors and Associated Equipment, 2011. 654(1): p. 250-257.

7. Wilson, M., et al., A $10 \mathrm{~cm} \times 10 \mathrm{~cm}$ CdTe Spectroscopic Imaging Detector based on the HEXITEC ASIC. Journal of Instrumentation, 2015. 10(10): p. P10011.

8. Boone, M.N., et al., High spectral and spatial resolution X-ray transmission radiography and tomography using a Color X-ray Camera. Nuclear Instruments and Methods in Physics Research Section A: Accelerators, Spectrometers, Detectors and Associated Equipment, 2014. 735: p. 644-648.

9. Scuffham, J., et al., A CdTe detector for hyperspectral SPECT imaging. Journal of Instrumentation, 2012. 7(08): p. P08027.

10. Pani, S., et al. Optimization of K-edge subtraction imaging using a pixellated spectroscopic detector. in Nuclear Science Symposium and Medical Imaging Conference (NSS/MIC), 2012 IEEE. 2012. IEEE.

11. Egan, C., et al., 3D chemical imaging in the laboratory by hyperspectral X-ray computed tomography. Scientific reports, 2015. 5: p. 15979.

12. Egan, C.K., et al., Dark-field hyperspectral X-ray imaging. Proceedings of the Royal Society A: Mathematical, Physical and Engineering Science, 2014. 470(2165): p. 20130629. 
13. Bourgeois, D., et al., Micro-distribution of uranium in bone after contamination: new insight into its mechanism of accumulation into bone tissue. Analytical and bioanalytical chemistry, 2015. 407(22): p. 6619-6625.

14. Jacques, S.D.M., C.K. Egan, M.D. Wilson, M.C. Veale, P. Seller, and R.J. Cernik, A laboratory system for element specific hyperspectral X-ray imaging. Analyst, 2013. 138(3): p. 755-759.

15. Liotti, E., et al., Mapping of multi-elements during melting and solidification using synchrotron X-rays and pixel-based spectroscopy. Scientific reports, 2015. 5: p. 15988.

16. Seller, P., et al. CdTe focal plane detector for hard x-ray focusing optics. in SPIE Optical Engineering+ Applications. 2015. International Society for Optics and Photonics.

17. Reiche, I., et al., Discovering vanished paints and naturally formed gold nanoparticles on 2800 years old Phoenician ivories using SR-FF-microXRF with the Color X-ray Camera. Analytical chemistry, 2013. 85(12): p. 5857-5866.

18. O'Flynn, D., et al., Explosive detection using pixellated X-ray diffraction (PixD). Journal of Instrumentation, 2013. 8(03): p. P03007.

19. O'Flynn, D., et al., Materials identification using a small-scale pixellated $x$-ray diffraction system. Journal of Physics D: Applied Physics, 2016. 49(17): p. 175304.

20. Mariscotti, M., A method for automatic identification of peaks in the presence of background and its application to spectrum analysis. Nuclear Instruments and Methods, 1967. 50(2): p. 309-320.

21. Blok, H., J. De Lange, and J. Schotman, A new peak search method for an automatic spectrum analysis program. Nuclear Instruments and Methods, 1975. 128(3): p. 545-556.

22. Likar, A. and T. Vidmar, A peak-search method based on spectrum convolution. Journal of Physics D: Applied Physics, 2003. 36(15): p. 1903.

23. Scuffham, J., M. Veale, M. Wilson, and P. Seller, Algorithms for spectral calibration of energyresolving small-pixel detectors. Journal of Instrumentation, 2013. 8(10): p. P10024.

24. Nielsen, N.-P.V., J.M. Carstensen, and J. Smedsgaard, Aligning of single and multiple wavelength chromatographic profiles for chemometric data analysis using correlation optimised warping. Journal of chromatography A, 1998. 805(1): p. 17-35.

25. Tomasi, G., F. van den Berg, and C. Andersson, Correlation optimized warping and dynamic time warping as preprocessing methods for chromatographic data. Journal of Chemometrics, 2004. 18(5): p. 231-241.

26. Katajamaa, M. and M. Orešič, Data processing for mass spectrometry-based metabolomics. Journal of chromatography A, 2007. 1158(1): p. 318-328.

27. Savorani, F., G. Tomasi, and S.B. Engelsen, icoshift: $A$ versatile tool for the rapid alignment of 1D NMR spectra. Journal of Magnetic Resonance, 2010. 202(2): p. 190-202.

28. Seller, P., et al., Pixellated $C d(Z n) T e$ high-energy X-ray instrument. Journal of Instrumentation, 2011. 6: p. C12009.

29. Veale, M., et al., Measurements of charge sharing in small pixel CdTe Detectors. Nuclear Instruments and Methods in Physics Research Section A: Accelerators, Spectrometers, Detectors and Associated Equipment, 2014.

30. Tomasi, G. http://www.models.life.ku.dk/dtw_cow.

31. Skov, T., F. van den Berg, G. Tomasi, and R. Bro, Automated alignment of chromatographic data. Journal of Chemometrics, 2006. 20(11-12): p. 484-497. 